\title{
Fault Diagnosis of Reducing Valve Based on Bispectra Slices
}

\author{
Wenbing $\mathrm{Wu}$ \\ Fuzhou Technical College of Foreign Studies, Fujian Fuzhou, 350202
}

\begin{abstract}
Bispectrum Includes nonsymmetric, nonlinear information of singals, it can be used to describe nonlinear phase couplings, especially quadratic phase couplings. Bicoherence function can evaluate the degree of quadratic phase couplings quantitatively, the bicoherence spectrum of normal and fault singals show different peaks characteristics. 2-D wavelet multi-level decomposition can compress the information of 2-D arrays while extracting features of frequences in primitive signals effectively. LSSVM can find the global optimization solution with small samples, so avoids local optimization, and it can reduce computation complexity. 26 groups of data obtained from the experiment were used to extract features by 2-D wavelet multi-level decomposition from bicoherence spectrum,then the features were input to the LSSVM, and diagnose the valve's faults, the correct rate is nearly $90 \%$, it is satisfactory.
\end{abstract}

Keywords-pressure relief valve; bicoherence spectrum; 2-D wavelet; LSSVM; fault diagnosis

\section{INTRODUCTION}

In mechanical fault diagnosis, often use a variety of signal processing methods, processing the vibration signal of machinery and equipment, to extract the fault characteristic information, in order to achieve the purpose of diagnosis of mechanical equipment failure. Power spectrum analysis is the most widely used, it gives the signal energy distribution of different frequency components, when mechanical equipment malfunction, the distribution of spectral energy is usually change, according to its to diagnose the mechanical fault.However, one of the biggest flaw of power spectrum analysis is that it does not contain the phase information between the frequency components, often can't handle the non-minimum phase system and non-gaussian signal, which is based on high order statistics of higher order spectrum can effectively deal with these problems, so as to improve the diagnostic accuracy of mechanical vibration fault. This paper based on obtain the pressure reducing valve vibration signal of bicoherence spectrum, in order to improve the efficiency of fault diagnosis using neural network, using 2-D wavelet multi-level decomposition on bicoherence spectrum to extract features.

\section{Pressure Reducing VAlve Hydraulic Circuit SYSTEM}

The pressure reducing valve of the experimental study is the forerunner type pressure reducing valve[1]. The oil through hydraulic pump to main lines, the maximum pressure of hydraulic pump to discharge oil from the overflow valve to adjust according to the needs of the main lines. When hydraulic cylinder needs pressure lower than the supply oil pressure of hydraulic pump, at this moment can string a pressure reducing valve in the oil outlet to relief pressure, pressure reducing valve can ensure constant pressure after decompression. Pressure reducing valve data acquisition system is shown in figure 1 .

When the pressure reducing valve has a foreign body in and out of the oil outlet, the pressure is high or low will affect the normal operation of the pressure reducing valve, in order to get the pressure reducing valve run signal in fault state, in this paper, the experiments special set up in the pressure reducing valve the oil inlet and outlet at the same time plus $\phi$ $3 \mathrm{~mm}$ iron core fault, through the experiment can simulate working the fault condition of pressure reducing valve.

\section{DATA ACQUISITION AND DYNAMIC TESTING PROGRAM}

Vibration signal acquisition and processing software is virtual instrument software development tools LabVIEW [2] Using LabVIEW and PCI - 6014 data acquisition card and an acceleration sensor, in turn to collect speed regulating valve under normal and fault condition of the vibration signal. In the process of test, the sampling frequency is set to $1024 \mathrm{~Hz}$, reads the frequency is $512 \mathrm{~Hz}$. In the state of each signal measurement, let the oil pressure from 1MPA 5 MPA divide into five classes. The sampling process time is about $2 \mathrm{~min}$. The quantity of data used in this paper experiment is 512

Due to in the process of testing the influence of external and internal factors of the system inevitable in the process of the output with many unwanted ingredients, this article used the median method to the collected vibration signal preprocessing.

\section{Bicoherence SPECTRUM}

Any gaussian process of high-order cumulant is zero, in theory can completely inhibit the effect of colored gaussian noise [2]. Assuming that high-order cumulant $c_{k x}\left(\tau_{1}, \cdots, \tau_{k-1}\right)$ is absolutely summable:

$$
\sum_{\tau_{1}=-\infty}^{\infty} \ldots \sum_{\tau_{k-1}=-\infty}^{\infty}\left|c_{k x}\left(\tau_{1}, \cdots, \tau_{k-1}\right)\right|<\infty
$$

$\mathrm{K}$ order cumulant spectrum is defined as the $\mathrm{k}$ order cumulant of the $\mathrm{k}-1$ dimensional Fourier transformation namely: 


$$
\begin{aligned}
& S_{k x}\left(\omega_{1}, \cdots, \omega_{k-1}\right) \\
= & \sum_{\tau_{1}=-\infty}^{\infty} \cdots \sum_{\tau_{\mathrm{k}-1}=-\infty}^{\infty} \mathrm{c}_{\mathrm{kx}}\left(\tau_{1}, \cdots, \tau_{k-1}\right) \exp \left[-j \sum_{i=1}^{k-1} \omega_{i} \tau_{i}\right]
\end{aligned}
$$

High-order cumulant spectrum is often referred to as high order spectrum, the third order spectrum (also known as bicepstrum) expression is:

$$
B_{x}\left(\omega_{1}, \omega_{2}\right)=\sum_{\tau_{1}=-\infty}^{\infty} \sum_{\tau_{2}=-\infty}^{\infty} c_{3 x}\left(\tau_{1}, \tau_{2}\right) e^{-j\left(\omega_{1} \tau_{1}+\omega_{2} \tau_{2}\right)}
$$

AR model is established after the bispectrum can also be used in the form of frequency characteristic function to express:

$$
B\left(\omega_{1}, \omega_{2}\right)=\gamma_{3 a} H\left(\omega_{1}\right) H\left(\omega_{2}\right) H^{*}\left(\omega_{1}+\omega_{2}\right)
$$

Among them,

$$
H(\omega)=\frac{1}{1+\sum_{i=1}^{p} \phi_{i} e^{-j i \omega}}
$$

is frequency

characteristic function, $\gamma_{3 a}=E\left\{a^{3}(n)\right\}$ is skewness, a(n) is an independent and identically distributed non-gaussian random process, $\mathrm{p}$ for the order of AR model.

Bispectrum contains the information asymmetric and nonlinear signal, can be used to describe the nonlinear phase coupling, especially the quadratic phase coupling, but because bispectrum and the signal are related to the power spectrum which in $\omega_{1}, \omega_{2}, \omega_{1}+\omega_{2}$, in the description of the fault asymmetric and nonlinear characteristics, sometimes the effect is not very obvious and intuitive.Therefore, in order to effectively reflect the characteristics of the bispectrum, often

let bispectrum use the power spectrum in $\omega_{1}, \omega_{2}, \omega_{1}+\omega_{2}$ module the normalization processing,get the bicoherence function [3].Bicoherence function can be quantitative estimates of quadratic phase coupling of coupling degree. Its physical meaning is: signal in the angular frequency $\omega_{1} \omega_{2}$ of the nonlinear phase coupling energy of signals in $\omega_{1}+\omega_{2}$ the proportion of the total energy.Bicoherence function quantitatively describe the system quadratic coupling degree. bicoherence function expression is shown in the following type:

$$
B_{c}\left(\omega_{1}, \omega_{2}\right)=\frac{B\left(\omega_{1}, \omega_{2}\right)}{\sqrt{\left|P\left(\omega_{1}\right) P\left(\omega_{2}\right) P\left(\omega_{1}+\omega_{2}\right)\right|}}
$$

Among them, $P(\omega)$ is power spectrum, and $P(\omega)=\gamma_{2 a}|H(\omega)|^{2} ; \quad \gamma_{2 a}=\sigma_{a}^{2}$ is non- gaussian white noise the variance of $a_{t}$.

\section{V.ANALYSIS OF EXPERIMENTAL RESULTS}

\section{A. Bicoherent Spectra Analysis}

First by experimental data obtained normal signal and bicoherence spectra of fault signal, the bicoherence spectra is actually a $2 \mathrm{D}$ array of $3 \mathrm{D}$ graphics.In this paper, for each group of data obtained using 512 data points to analyze, and the bicoherent spectrum obtained is $255 * 2552 \mathrm{D}$ array, this paper selected oil pressure is 1 MPA, 3 MPA, 5 MPA measured two vibration condition of the three sets of data, the normalized after the $3 \mathrm{D}$ graphics for bicoherent spectrum is shown in figure 2 and figure 4.Figure in the horizontal axis shows the frequency, the unit for HZ, vertical axis shows after normalized amplitude of the size, no dimension.Can be seen from figure 2 and figure 4 [4-7], regardless of hydraulic valve in normal condition or fault condition, spectral peak are present on bicoherence spectra, its gaussian deviation is obvious. In the above figure bicoherent spectrum amplitude exists and is not zero, clear that the vibration signals of the hydraulic valve with nonlinear and non-gaussian. For nonlinear system, in some frequency will show strong correlation, namely between the two frequency component and the spectrum value occurred quadratic phase coupling, its performance is under the coordinates of double frequency bicoherent spectral appear higher spectrum peaks on the $3 \mathrm{D}$ graphics. Under normal condition, the bicoherent spectrum of quadratic phase coupling presented the phenomenon of a broader extension and distribution more uniform, and in the double frequency coordinate spectrum peaks in relatively flat and concentrated, in the case of failure, appeared the more spectral peak on bicoherent spectrum diagram, at the same time in some frequency, showed a strong nonlinearity, and the shape of the spectral peak and energy have certain differences, spectrum peaks showed miscellaneous and disorder. According to the normal signal and fault signal of in dual chromatogram intuitively, can consider to fault diagnosis by bispectrum.

\section{B. Bicoherent Spectrum Feature Extraction}

Wavelet transform is a kind of signal time -- scale analysis methods, it has the characteristics of multi-resolution analysis, and in both time and frequency domain has the ability of denoting local signal characteristics, is a kind of fixed window size but the shape variable, time window and frequency window variable time-frequency localization analysis method. Two-dimensional wavelet function is through a one-dimensional wavelet function after tensor product of transformation, the two-dimensional wavelet function decomposition is low frequency part of the scale $j$ is decomposed into four parts: low frequency part of the scale $\mathrm{j}+1$ and the high frequency part of three directions (horizontal, vertical and diagonal lines) ${ }^{[8-9]}$

Based on the fault identification, if directly using the bispectrum data obtained input the neural network, one is the computational cost is too big, the second is acquired through experiments the results are not ideal. Due to in the original signal included various frequency information must be response to its high order spectrum, and wavelet 
decomposition can effectively extract the frequency information, so this paper uses the two-dimensional wavelet decomposition of the measured data in each group of bicoherent spectrum for feature extraction, based on the symmetry of the bicoherent spectrum, this article uses the db1 wavelet. Through experimental comparison and analysis, after the original data was decomposed by the two-dimensional wavelet function, and when extract the scale for 5 of 5 level low frequency coefficient of compression as LSSVM input vector, the best recognition results have been achieved, the low frequency coefficient of compressed is $8 * 8$ two-dimensional array, after normalized the low frequency coefficient its graph is shown in corresponding data of beneath the bicoherence spectra, as shown in figure 3 and figure 5. In figure 3 and figure $5 \mathrm{x}, \mathrm{y}$ axis respectively represent compressed the dimensions of the two-dimensional matrix, $\mathrm{z}$ axis represents the eigenvalues of extraction, dimensionless.

\section{The Fault Identification}

Due to the least squares support vector machine (SVM) solve the problem better such as the small sample, nonlinear, high dimension, local minimum point and so on, can get the global optimal under limited samples, so as to avoid local optimal problem, and has the advantages of lower computational complexity
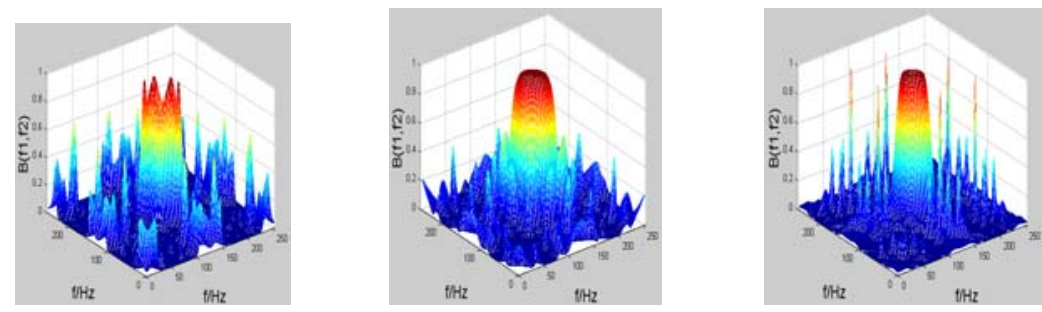

FIGURE I. NORMAL SIGNAL BICOHERENT SPECTRA
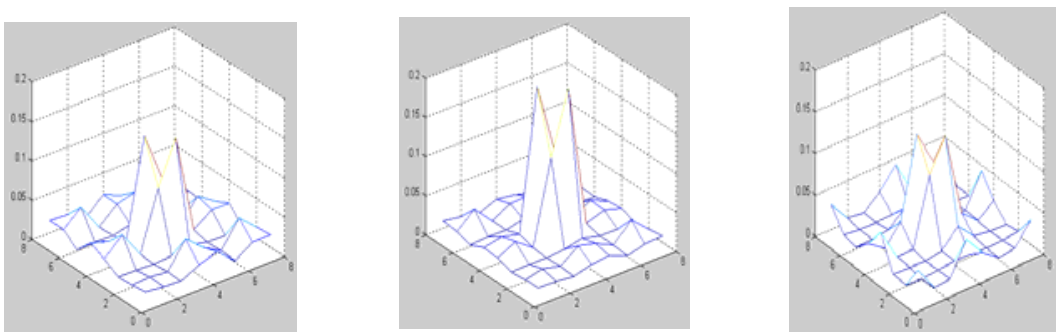

FIGURE II. NORMAL SIGNAL 5 LAYER WAVELET LOW FREQUENCY COMPRESSION DIAGRAM
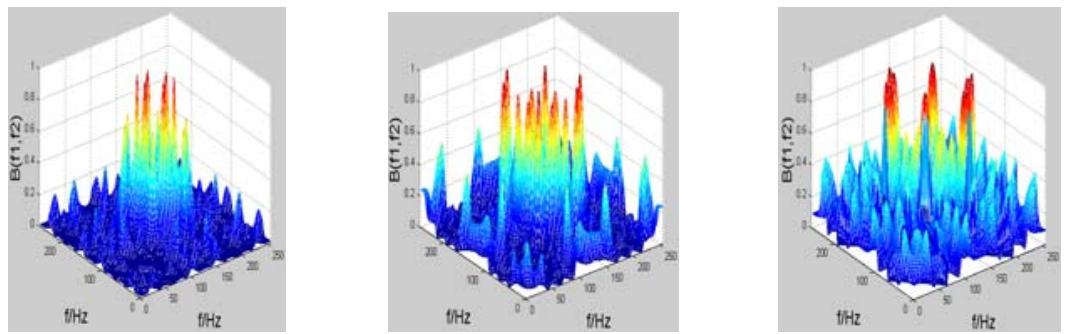

FIGURE III. FAULT SIGNAL BICOHERENCE SPECTRA
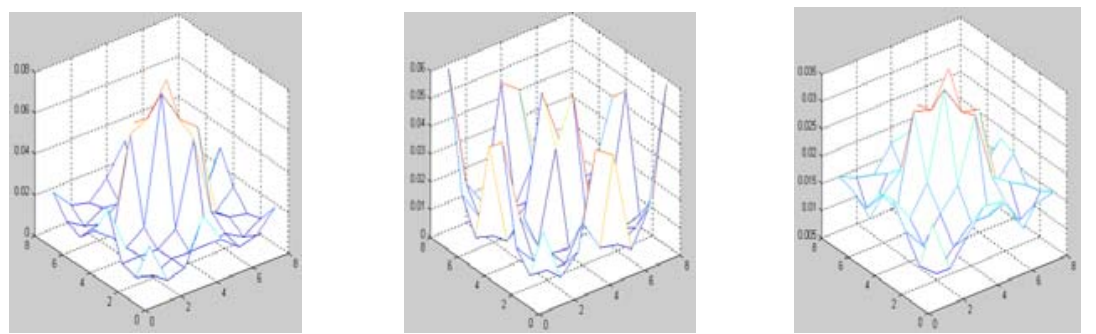

FIGURE IV.FAULT SIGNAL 5 LAYER WAVELET LOW FREQUENCY COMPRESSION DIAGRAM 
TABLE I. THE NORMALIZED EIGENVALUE TABLE

\begin{tabular}{|c|c|c|c|c|c|c|c|c|}
\hline normal & 0.0062 & 0.0032 & 0.0020 & 0.0130 & 0.0217 & 0.0009 & 0.0151 & 00000 \\
\hline \multirow[b]{2}{*}{ state } & 0.0235 & 0.0044 & 0.0111 & 0.0453 & 0.0403 & 0.0042 & 0.0161 & 。 \\
\hline & 0.0027 & 0.0080 & 0.0016 & 0.0051 & 0.0104 & 0.0017 & 0.0092 & 000000 \\
\hline \multirow{2}{*}{ fault } & & & $0 \circ$ & 00154 & 00127 & 00036 & 00098 & \\
\hline & 0.0091 & 0.0041 & 0.0043 & 0.0119 & 0.0168 & 0.0032 & 0.0125 & \\
\hline \multirow{2}{*}{ state } & 0.0117 & 0.0057 & 0.0089 & 0.0114 & 0.0102 & 0.0116 & 0.0102 & 000000 \\
\hline & \multicolumn{8}{|c|}{000000} \\
\hline
\end{tabular}

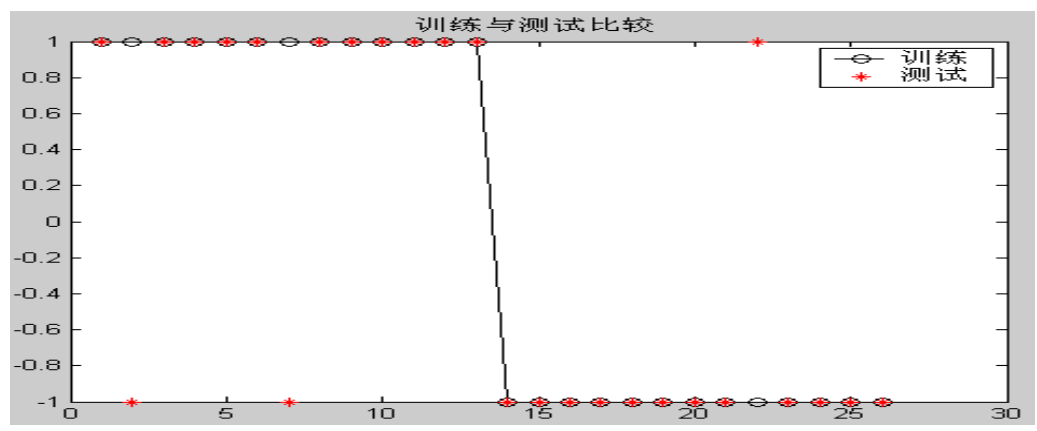

FIGURE V. SIMULATED RESULT GRAPH

This paper according to the literature (10), established the same LSSVM. Due to the radial basis kernel function is the ability to learn more, this article selects the function of pressure reducing valve fault identification, the function expression is:

$$
K\left(x_{i}, x\right)=\exp \left\{-\frac{\left|x_{i}-x\right|^{2}}{2 \sigma^{2}}\right\}
$$

This experiment in five different pressure cases 18 sets of normal data and 18 sets of fault data were measured respectively, in training LSSVM, low frequency coefficient of compression after the normalization processing, method is to use a $8 * 8$ of two-dimensional array of each data divided by the two-dimensional array of the sum of all the data values, after the normalization of data shown in table one. Then respectively make the normal state and failure state code is 1 and -1 , training respectively in the table one of normal data and fault data to retrieve the corresponding 5 sets of data altogether 10 groups, to set up by the least squares support vector machine (SVM) training, after training, recycling the support vector machine (SVM) is used to identify the fault, after parameter optimization, take punishment factor $\gamma=79$, the radial basis kernel function parameter $\sigma^{2}=0.71$,using after training the network pattern recognition with the remaining 26 sets of data, as shown in figure 6,26 sets of data the state after the simulation shown in figure, the hollow circle said training data pre-set state, such as the first 13 sets of data is measurement of normal data, which should be in figure the above horizontal line represents 1 state, post 13 sets of fault data should be the next horizontal line represents -1 state. red solid circle said after LSSVM simulation to data of classification results, if a set of data the hollow circle and red solid circular overlap, indicates the simulation results of the set of data is correct, otherwise the error. Results show that in the normal data only 2 groups, in the failure data only 1 group identification errors, the general recognition accuracy is close to $90 \%$ (excluding 10 sets of training data), shows that this paper proposed fault diagnosis method is feasible.

\section{The CONCLUSION AND PROSPECT}

Bispectrum contains the information asymmetric and nonlinear signals, can be used to describe the nonlinear phase coupling, especially the quadratic phase coupling. Bicoherence function can be quantitative estimates of quadratic phase coupling of coupling degree. The normal signal and fault signal of the pressure reducing valve of bicoherent spectrum presented different wave characteristics. In order to improve the accuracy of fault diagnosis and reduce the computational complexity, at the same time considering the wavelet decomposition can effectively extract the frequency information in the original signal, in this paper using two-dimensional wavelet multistage decomposition to two-dimensional array of bicoherent spectral information compression, considering the least squares support vector machine (SVM) can get the advantages of the global optimal under limited samples, this paper using compressed coefficient of low frequency as the input of LSSVM for fault diagnosis of the pressure reducing valve, has obtained the good effect. Due to the high-order spectrum can effectively remove gaussian noise, the author will be in the follow-up research work, compare the fault diagnosis method with other methods, to determine its advantages, and analyze the reasons.

\section{ACKNOWLEDGMENT}

This paper is supported by National Natural Science Foundation of China(61562063)

Key science and technology project of Jiangxi Provincial 
Education Department(GJJ161234)

Author introduction: wu wenbing (1968--), male, Phd in electronic, vice professor

\section{REFERENCES}

[1] li-ping zhang. Hydraulic valve principle, use and maintenance [M]. Beijing: chemical industry press, 2005:146,155,204,147-148.

[2] xian-da zhang. Modern signal process [M]. Tsinghua university press, 1995.

[3] Yang jiang tian, jin-wu xu. Bicoherent spectrum characteristics of rotating machinery fault and its recognition[J]. Journal of mechanical engineering, 2000, 36 (09) : 103-106.

[4] Peng Zhijun yi-jian huang. Overflow valve fault diagnosis based on AR bispectrum [J]. Journal of mechanical science and technology, 2007, 26 (07) : 908-912.

[5] Chen Bingsan, Huang Yijian, Cai Qizhi. Study on autoregressive trispectrum slices [C], Chinese control and decision conference, Yantai, 2008:5124-5129.

[6] Cai Jizhi yi-jian huang. Three auto-regressive spectral slicing study [J]. Journal of instruments and meters, 2009, 30 (2) : 1-6.

[7] Gang CHEN, Yang, LIU, Wen - an ZHOU. Research on intelligent fault diagnosis -based on time series analysis algorithm [J]. The Journal of China Universities of Posts and Telecommunications, 2008,15 (1) : 68-74.

[8] chang-hua hu, Zhang Junbo NieJun. Based on the MATLAB system analysis and design - wavelet analysis $[\mathrm{M}] \mathrm{xi}$ 'an: xi 'an university of electronic science and technology press, 1999.12 53-125

[9] wei-li gong,li chen, Multi-scale anisotropic characteristics of the coal and rock structure SEM image analysis, geotechnical mechanics and engineering journal, 2010 (5)

[10] Wu Wenbing,yi-jian huang. Hydraulic valve fault signal detection based on AR parameters. Heavy machinery, 2011 (2): 15-18. 